\title{
Priorities, Technology, \& Power: Co-Designing an Inclusive Transit Agenda in Kampala, Uganda
}

\author{
Lynn Kirabo \\ lkirabo@andrew.cmu.edu \\ Carnegie Mellon University \\ Pittsburgh, PA \\ Devon Barry \\ devonb@andrew.cmu.edu \\ Carnegie Mellon University \\ Pittsburgh, PA
}

\author{
Elizabeth Jeanne Carter \\ ejcarter@andrew.cmu.edu \\ Carnegie Mellon University \\ Pittsburgh, PA
}

Aaron Steinfeld

steinfeld@cmu.edu

Carnegie Mellon University

Pittsburgh, PA

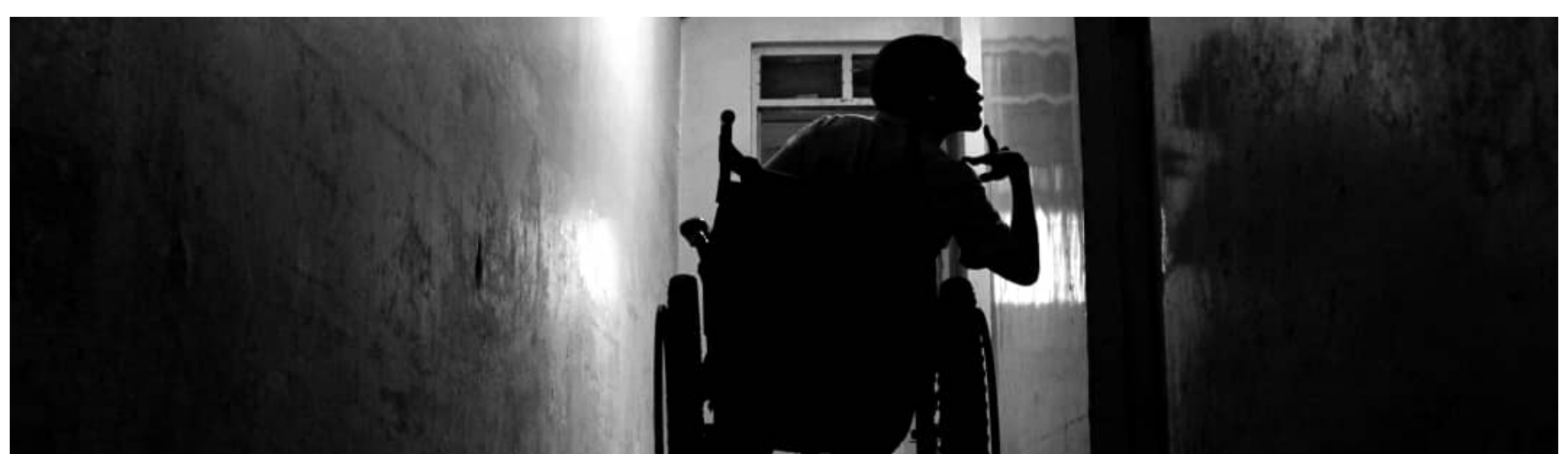

Figure 1: A person who uses a wheelchair strikes a pose in a narrow hallway in Kampala. Image courtesy of Zahara Abdul.

\section{ABSTRACT}

There is considerable effort within the HCI community to explore, document, and advocate for the lived experiences of persons with disabilities (PWDs). However, PWDs from the Global South, particularly Africa, are underrepresented in this scholarship. We contribute to closing this gap by investigating the unmet transit needs and characterization of technology within the disability community in Kampala, Uganda. We investigated transportation due to the increase in ride-share solutions created by widespread mobile computing and the resulting disruption of transportation worldwide. We hosted co-design sessions with disability advocates and adapted the stakeholder tokens method from the value-sensitive design framework to map the stakeholder ecosystem. Our key insight is the identification of a new group of non-traditional core stakeholders who highlight the values of inclusion, mobility, and safety within the ecosystem. Finally, we discuss how our findings engage with concepts of disability justice and perceptions of power.

This work is licensed under a Creative Commons Attribution International 4.0 License.

CHI '21, May 8-13, 2021, Yokohama, Japan

(c) 2021 Copyright held by the owner/author(s)

ACM ISBN 978-1-4503-8096-6/21/05.

https://doi.org/10.1145/3411764.3445168

\section{CCS CONCEPTS}

- Human-centered computing $\rightarrow$ Empirical studies in accessibility; - Social and professional topics $\rightarrow$ People with disabilities.

\section{KEYWORDS}

Power Dynamics, People with Disabilities, Accessibility, ValueSensitive Design, Stakeholder Tokens Method, Uganda, Kampala, Global South

\section{ACM Reference Format:}

Lynn Kirabo, Elizabeth Jeanne Carter, Devon Barry, and Aaron Steinfeld. 2021. Priorities, Technology, \& Power: Co-Designing an Inclusive Transit Agenda in Kampala, Uganda. In CHI Conference on Human Factors in Computing Systems (CHI '21), May 8-13, 2021, Yokohama, Japan. ACM, New York, NY, USA, 11 pages. https://doi.org/10.1145/3411764.3445168

\section{INTRODUCTION}

Under-representation in research scholarship and populations has diverse effects on artifacts and the future of research fields. The human-computer interaction (HCI) community has notably lacked representation in both scholarship $[18,19]$ and populations from African countries, particularly for individuals with disabilities. This double lack of representation can lead to the rise of solutions and findings that reveal dissatisfaction, cost mismatches, and unique appropriations to match contextual circumstances. According the 
United Nations, there are 80 million people on the African continent who have a disability [38]. In Uganda specifically, that number is nearly 1.4 million [51, p. 4]. It is high time that these populations are included in conversations about technology that could impact their way of living.

Design and research movements are unanimous about the importance of inclusion with specific regard to physical environments [62] and technological spaces [33, 39, 65]. However, these approaches often fail to adapt to local contexts in the Global South, including countries like Uganda, due to social-cultural differences. This issue presents the need for design movement agendas that engage with both the notion of culture and that of inclusion. One example of such a movement is included in the principles that govern the Value-Sensitive Design framework [15]. The Value-Sensitive Design framework advocates for prioritizing human values across multiple technology stakeholders. In this framework, human values represent inherent beliefs and concepts of dignity and fairness that are culturally relevant to local contexts. Situating these values across multiple stakeholders reveals nuances that technology designers and researchers should engage and grapple with as they propose methodologies and interventions. Relatedly, another example of a movement with similar principles is the Disability Interaction (DIX) Manifesto [24]. The principles of "co-created solutions" and "value use and usefulness" particularly advocate for the amplification of the voices of persons with disabilities.

We extend this scholarship by applying these methodologies to assistive technology contexts in the Global South (specifically, Kampala, Uganda). In this work, we use qualitative methodologies such as interviews and design exercises to investigate the unmet transit needs of persons with disabilities in Kampala, Uganda. We chose transportation given its embedded nature in daily lived experiences. Furthermore, there is the steady and continued proliferation of rideshare solutions that use mobile computing to provide both transit and supplementary services (e.g., package delivery). We adopted the Stakeholder Tokens Method [66] from the Value-Sensitive Design framework to co-design a map that represents stakeholders within the disability ecosystem and the relationships that exist between them. It is important to understand who the different stakeholders are and the influence they hold, especially when designing new interventions.

This work makes three contributions to the HCI community. Firstly, it engages and amplifies the voices of traditionally underrepresented populations in the research community. Technology solutions not only need to be implemented with consideration of different contexts, but also to be sensitive to how they impact different power dynamics. Secondly, it identifies a new group of stakeholders in this domain and context that highlight the values of inclusion, mobility, and safety within the ecosystem. This affirms the importance of identifying the different perspectives in an ecosystem. Lastly, this work presents an invitation to explore an inclusive transit agenda through offering practical applications for HCI researchers and practitioners. We provide a guide for how to identify transit stakeholders in unique contexts and identify opportunities for future research in this domain.

\section{RELATED WORK}

The continued proliferation of mobile technologies in the Global South has given rise to contributions at the intersection of technology and transportation [1, 14, 29, 35, 47, 48, 58, 59]. Mobile technologies such as mobile phones have also been appropriated as assistive devices that bridge physical barriers [6] and promote inclusivity within personal networks [5]. We scope this related work section to review literature in three categories: general assistive technologies, accessible transportation, and lived experiences. In each of these categories, we start by presenting a broad picture of work in the Global South and then narrow it to research specific to Uganda, when available.

\subsection{The Investigation into Assistive Technologies: Domains and Applications}

There is some debate over the affordability and practicality of assistive technologies such as optical character recognition tools, speech recognition, screen readers, braille keyboards, etc., in Global South contexts. Pal et al. [54] critiqued these technologies for persons with visual impairments. They cited the exorbitant costs of these tools and their lack of applicability to local context. They argued that this gap between availability and need can be filled through promoting accessibility scholarship in the Global South and by encouraging the creation of local tools made by people from local contexts.

However, before advocating for locally made technologies, it is necessary to disseminate knowledge about the importance of creating inclusive environments. In Uganda, several scholars [4, 46] found that government websites violated accessibility standards, thus denying users with visual impairments access to online services and information. Baguma et al. [4] specifically surveyed local web developers and found that, even though they were aware of accessibility guidelines, there were still barriers that prevented implementation. Scholars have also investigated the use of assistive technologies to create inclusive educational environments for children with disabilities [3] who are likely to drop out of school because of their disability. They specifically advocated for the inclusion of information communication technologies (ICTs) as aids in the teaching experience. Kearney et al. [31] took a multiple modality approach by incorporating both computers and physical tactile objects in their curriculum designed to teach the basics of JavaScript, HTML, and CSS to blind and low vision students in Gulu, northern Uganda. Using a variety of materials, including a tactile board game, students with no previous exposure to programming were able to make interactive websites. They emphasized the importance of contextualizing learning to account for students' previous exposure to assistive technologies such as screen readers. They also noted the importance of designing for situations that may be unique to a local context, such as utility outages or low internet accessibility, as well as considering cultural norms. This research highlights the value of understanding both the local contexts within different ecosystems and the motivations of different stakeholders. We extend this scholarship by co-designing a map that represents stakeholders within the disability ecosystem and exploring the values within the different relationships. 


\subsection{Through the Lens of Accessible Transportation: Online Maps \& Ride-Share Applications}

Around the world, mainstream technologies, such as online maps and ride-share applications, have been used to improve mobility. When examined through the local context of the Global South, interesting insights emerge. For instance, Google Maps is not considered an efficient navigation tool for persons with visual impairments in India [26]. Designed for a specifically organized setting (i.e., the Global North), it is not readily applicable to urban settings in the Global South that do not follow grid layouts. Also, ride-share technology in India is characterized as "a socio-technical collaborative effort" that includes the rider with a disability, a driver, and technology [25, p. 85:3]. This collaborative effort gives rise to the notion of a nuanced idea of independence where riders can travel independently, but rely on drivers where necessary.

Similarly in Uganda, Kirabo et al. [34] found that persons with disabilities resorted to using ride-share applications to escape the social barriers tied to traditional forms of transportation. They discussed how the discrimination and harassment of persons with disabilities while accessing traditional public transportation in Kampala and Kigali was a motivating factor for their adoption of rideshare technologies. The participants recounted instances of being left by the road side or being asked to pay double the typical fare because of their assistive devices. The participants also noted how these experiences left them feeling like second-class citizens within their communities. Ride-share applications reduced these occurrences. This work shows evidence of technologies improving transit experiences; however, they can only be used by those with access to both a smartphone and internet. We further this research by discussing new opportunities in which ride-share companies can engage with various stakeholders in the disability ecosystem to foster more inclusive experiences for persons with disabilities.

\subsection{The Importance of Understanding Lived Experiences of Persons with Disabilities}

The discussion of who has the right to be included in the design process is critical, especially when working with traditionally disenfranchised communities. Scholars [56, 64] have used co-design methods to give more communities voices in problem definition and solution creation. The exploration of lived experiences is a specific avenue that provides rich insight into values and everyday occurrences that can sometimes be overlooked in evaluation studies. Extending work at the intersection of critical race and feminist theory, Pal et al. [55] sought to understand the experiences of women with disabilities in Malawi and Rwanda. In a survey conducted among people with visual impairments in the capital cities of Blantyre and Kigali, they found similar narratives of marginalization faced by women with disabilities in both cities. They noted how social exclusion impeded kinship bonds between women with disabilities and their communities. Furthermore, they discussed how technology unwittingly is a double-edged sword by promoting aspirations while contributing to marginalization. Moreover, $\mathrm{Pal}$ and Lakshmanan [53] observed this contrary impact of assistive technologies in other regions. On the one hand, it impacted participant aspirations, specifically for career goals. However, participant narratives also revealed employment-related issues among the first generation of "assistive technology users" in Bangalore, India. In their interviews, participants gave examples of misalignment between the tasks assigned and ability, the lack of employment offers for more technically qualified candidates, and underpayment when they eventually succeeded in getting jobs. These descriptions of lived experiences give insights into the perceptions of stakeholders. We continue this tradition of eliciting lived experiences through conversations about the power dynamics that exist within the disability ecosystem

\section{METHODOLOGY}

\subsection{Study Design \& Data Collection}

We conducted 6 online semi-structured interviews, each with 2 design exercises. Our goal was to elicit the unmet needs of the disability community and characterize the current and future roles of technology. First, participants completed a design exercise to identify problem areas in transportation and disability and envision possible technological solutions. In our second activity, we invited our participants to co-design a stakeholders' map that revealed underlying power structures within the disability ecosystem. All interviews were conducted over Skype. Given the constraints of an online study, we organised the activities to lessen the time and cognitive burden on our participants. This research was approved by our university's Institutional Review Board as well as by university faculty in Kampala.

We recruited our participants using social media. We invited adults who work in organisations that advocate for and work with people with disabilities to participate. Our assumption was that members of this group interacted with multiple parts of the disability ecosystem. We selected participants after the research team verified participant roles in organisations. This was solely based on the disability type and nature of the organisation. We wanted to speak to people with diverse experiences (Table 1). After participating in the study, participants were compensated $\$ 40$ (the average local cost of 1 month of mobile data) for their time using mobile money transfer.

3.1.1 Design exercise 1: Waving a magic wand. Our first design exercise was inspired by previous work [16]. We adapted the exercise for our context by removing magic because it can conjure associations with the occult that might make participants uncomfortable depending on their upbringings and beliefs. In this exercise, we asked participants to imagine what challenges they would solve about public transportation if they were given all the money they needed. Participants were given time to reflect, write down, and share their answers with the interviewer. Then, using the HowMight-We method [12], we probed whether technology might be used to address the challenges raised. We also asked questions to elicit further characterizations of the roles technology presently plays in accessibility and transportation (i.e., in raising awareness, reducing stigma and increasing access to services). Lastly, participants were asked to identify factors they felt were critical to the design of inclusive technology. 
Table 1: Participant Information. Participant names have been changed to protect their identities.

\begin{tabular}{cccl}
\hline Name & Disability & Organisation & Experience \\
\hline Kagenza & Deaf/Hearing Loss & Disability NGO: Education Focus & $>10$ years \\
Muwonge & Mobility Impairment & Government & $>10$ years \\
Katongole & Mobility Impairment & Disability NGO: Arts Focus & $1-5$ years \\
Nassiwa & Albinism/Low Vision & Disability Activist & $1-5$ years \\
Kunda & None & Sign Language Interpreter & $1-5$ years \\
Murungi & None & NGO: Internally Displaced Peoples & $<1$ years \\
\hline
\end{tabular}

3.1.2 Design exercise 2: Map generation. We employed the stakeholder tokens method (adapted for an online study) [66] to codesign a stakeholders map. Our use of co-design is predicated on invitation and empowerment of non-designer users to contribute to the creation process $[67,68]$. We were interested in creating a space where participants contributed through their own lived experiences. The stakeholder tokens method from the Value-Sensitive Design Framework [15] can be used to reveal complex sociopolitical relationships among different stakeholders. We developed an initial image of nodes (i.e., stakeholders) using existing literature about the disability community [2, 7, 9, 11, 40, 43, 57] and local knowledge. This was guided by these questions: Who are the people, communities, and groups involved with persons with disabilities? There were no connections drawn between any nodes.

This document was piloted with three local Ugandan researchers who work with vulnerable populations in Uganda. These researchers were asked whether the image was a representation of the current disability ecosystem. Over these iterations, 11 new nodes were added to the final map that was used with participants in the study. The final image consisted of 22 nodes: Veterans, Aid Partners, Person with Disability, Hospitals, Bus Drivers, Local City Authority, Boda Boda Drivers, Activists, Rehabilitation Centers, Community Health Workers, Technology Startup Community, Ministry of Health, Metropolitan Police, School Teachers, Pedestrians, Person with Disability's Family, Bicycle Operators, Traffic Police, Taxi Drivers, Industry Corporate Social Responsibility Teams, NonGovernmental Organisations, and the Ministry of Gender, Labour and Social Development (Figure 2A). The original stakeholder tokens method leverages the use of the tactile tokens [66]; our study was originally intended to mimic this by using a collaborative drawing tool. However, due to a diversity of screen sizes, resolutions and internet connections, we could not use the tool. Participants were sent the final version of the image over Skype and email in both JPG and PDF formats. They were asked if the image they were given was a complete representation of the disability ecosystem. Participants were asked to identify direct stakeholders, indirect stakeholders and entities they believed should not be on the map. Participants were also given the opportunity to add any stakeholders they thought were missing and asked to identify the relationships/connections that existed among the different stakeholders in the image. They were then encouraged to share scenarios that illustrated the various connections. The identified relationships among entities on the image were denoted by lines connecting them (Figure 2B). The goal of drawing these maps was to determine who participants understood the potential power brokers in the community to be as well as elicit any underlying values as described through the relationships drawn.

\subsection{Setting the Local Context}

Given that there are a wide variety of transportation methods and terms used around the world, we provide this section to describe typical Ugandan transit and colloquial language. This definition of terms allows us to follow the guidelines suggested in recent work [52] to maintain the voices and words used by our participants in our quotations. The vibrant city life of Kampala relies upon an informal network of privately owned vehicles that can be used by members of the general public for a fee. This informal system is not overseen by a government agency. Sidewalks are referred to locally as Pavements. We use this naming convention in this paper. Vehicles often pick passengers up at locations locally referred to as Stages. Vehicles include, but are not limited to:

- Boda boda: Motorcycles often have extra seat space behind the driver, which can be rented to passengers to go to their destinations in exchange for a fare. These motorcycles are named for their ability to travel efficiently from border to border and are particularly popular because of their ability to maneuver between cars that are stopped in traffic.

- Taxi: The Ugandan taxi is a 14-passenger van that carries riders between stages (pickup spots). They are staffed by a driver and a conductor who asks potential riders where they are going to see if they could reasonably join the existing journey and collects fares. Riders are not typically acquainted with each other, the driver, or the conductor.

\subsection{Data Analysis}

For our Magic Wand exercise, we used thematic analysis [8] to identify overlapping categories in participant responses. We created an aggregate map using the data from Design Exercise 2 (Figure 3). Due to incomplete data, one participant's map was not included in analysis. After combining the five maps, we noted the entities on the map that had no connections to other entities. We also noted instances where participants felt the need to justify the connection with example scenarios. Our map analysis led to the categorisation of stakeholders as Core or Periphery. We define Core stakeholders as entities that are who are central to the lived experiences of persons with disabilities. Periphery stakeholders are entities with incidental or compound (through other entities) relationships to persons with disabilities. We also use the term influential stakeholder to refer to entities that our participants perceived as power brokers within the disability ecosystem. There were some instances where Influential 

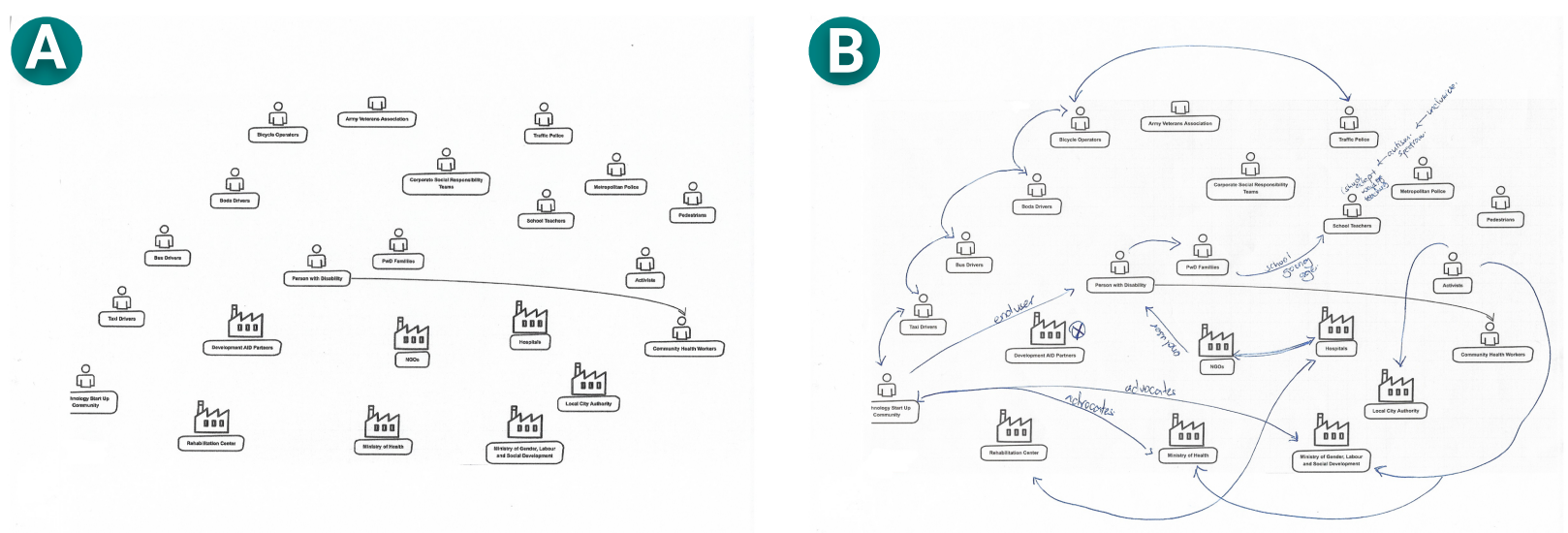

Figure 2: Image A (left) was the final image of nodes presented to the participants. Image B (right) is an example of a map with some connections drawn between nodes. Connections are denoted by lines.

stakeholders were also Core stakeholders; however, not all Core stakeholders were Influential.

\subsection{Limitations}

Due to the nature of online recruitment and participation, we acknowledge that our method limited participation to individuals who have both access to data connectivity and knowledge of online meeting tools. By limiting participation to only those individuals whose organisation affiliations could be proven, we acknowledge that this undoubtedly excluded grassroots activists who do not have any online presence and/or work in remote areas. Furthermore, while two participants mentioned travelling outside of Kampala for work, the experiences they shared were from Kampala. Nonetheless, we still believe that this work has important takeaways for growing urban populations (in Kampala and other Ugandan cities).

\subsection{Self Disclosure}

Data collection was conducted by the first author, who is a native of Uganda. This author has experience conducting research with communities in the Global South. The other authors are natives of North America. These authors have extensive experience working with communities of people with disabilities. One also has extensive experience in transportation research.

\section{FINDINGS}

\subsection{Participants' Wish List}

We present the participants' responses to the first design exercise, Waving a Magic Wand, below. In that exercise, they detailed how they would solve transportation challenges pertinent to the disability community if they were given unlimited resources. The participants' ideas are presented in the order of their preference of their priorities.
4.1.1 Disability awareness and understanding. Three participants mentioned the need to create programs that foster an awareness in the general public of the lived experiences of persons with disabilities. Participants reflected on the daily challenges of being confronted with apathy and blatant disregard by different members of the community. These behaviors were not limited to a specific social class or situation.
"...Even those who are educated have that stigma be- cause we do not understand what people are going through." - Murungi
"Change the attitude of transporters through their um- brella organisations: UTODA, Uganda Bus Owners As- sociations, Boda bodas have cooperatives, groups and associations. It can be the first step, because even if we bring accessible buses, if people still have the attitude towards people with disabilities they will still ignore us." - Kagenza

Additionally, Kunda noted that even when being asked for help, bystanders often acted like persons with disabilities were invisible. This further emphasized the need for a shift in the attitudes of people within the community, which one participant said cuts across existing social classes.

4.1.2 Transport infrastructure. During the sessions, three participants reflected on the state of the existing infrastructure such as roads, pavements, and stages, noting that they were often nonexistent or inaccessible. They explained that pavements are often shared with boda boda drivers who are trying to avoid traffic by riding on the pavement. They noted that it is then up to the pedestrian to carefully navigate their way, even on the pavement. This feat is challenging to people with disabilities that affect their sight and hearing. In other extreme cases, participants noted places where pavements did not exist. In these cases, pedestrians and vehicles are forced to share the same road (often to the annoyance of vehicle 


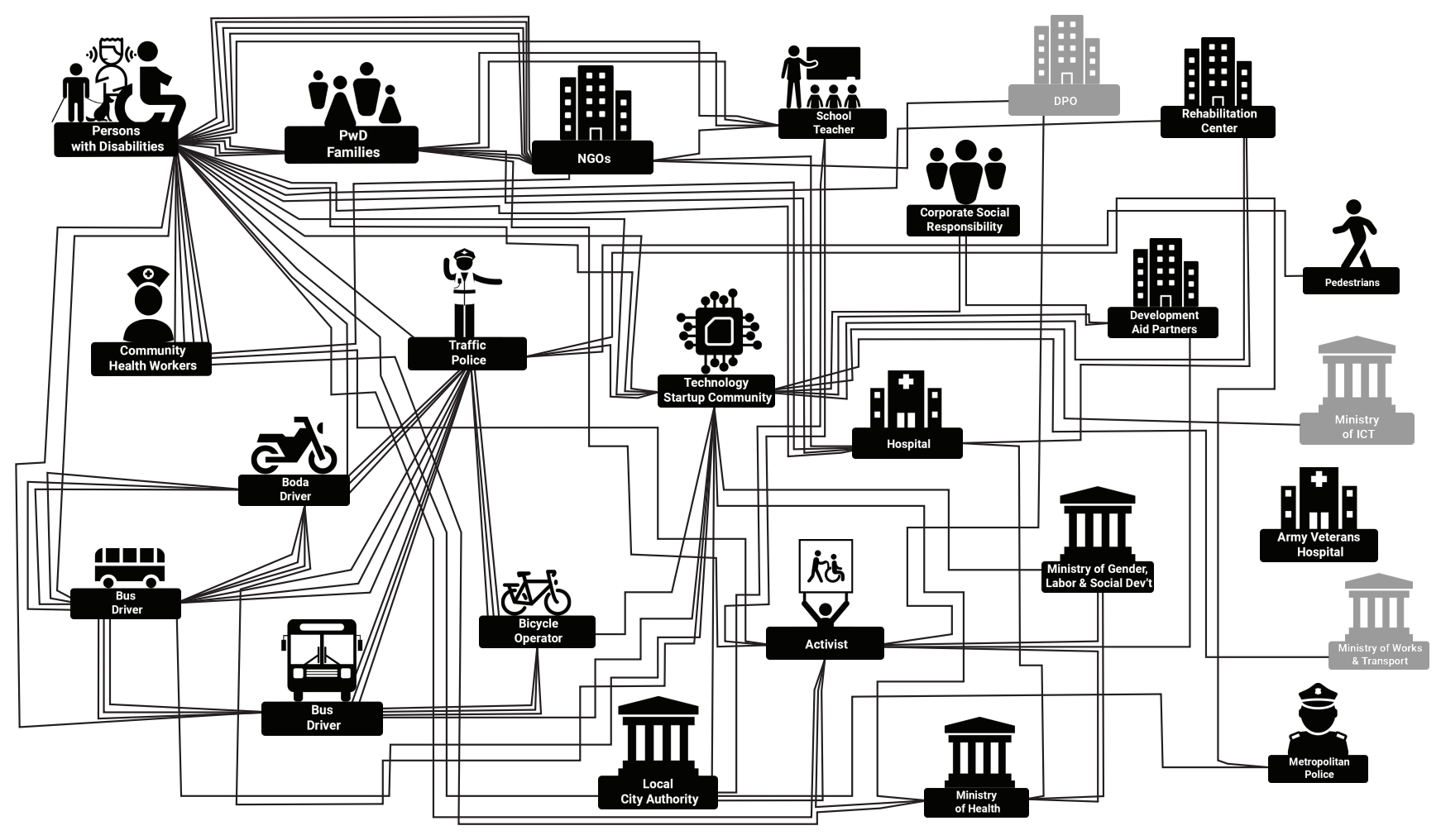

Figure 3: Stakeholder map showing the aggregate connections

drivers). In light of these challenges, participants advocated for investing their unlimited resources in building physical infrastructure that is accessible and safe.
"...the challenge of accessing public means, for example, it will require to ensure that we gazette properly these stages, in that people with disabilities can wait from there freely" - Katongole

4.1.3 A dignity-first agenda: Accessible vehicles. Three participants talked about investing some of the money in the purchase of accessible taxis, specifically citing the installation of ramps that can be used to board and disembark the vehicle. Katongole described that when using taxis, wheelchair users are normally carried into the vehicle by one of the transport operators (who is usually of no familial relation). The wheelchair is then stored in one of three places: the vehicle's boot (if available), tied to the top of the vehicle, or tied at the back of the vehicle. Katongole went further to explain the lack of dignity associated with how he was handled as well as the constant safety concern over how his property was stored.

4.1.4 Techno-futurism: Innovative assistive technology. Two participants also suggested using the money as an investment in specific technology ideas. Their rationale was that these ideas would support the daily commutes of persons with disabilities. Examples included smart embedded systems with a simple push button interface that would alert bus drivers of a blind passenger waiting for a taxi and audio controls on traffic lights. Muwonge specifically shared a dream of modifying his wheelchair to include a pumping mechanism that allows him to access vehicles with multiple heights.

\begin{abstract}
"I have a dream of a wheelchair that can raise up and go down. If it raises me up, I can enter a lorry or bus. Using my own wheelchair, I pump it and I go up. It will be raised and I am at that level of that vehicle then I can sit on it and then lower it and then go." - Muwonge
\end{abstract}

4.1.5 Diversity and under-representation. While most participants agreed that technology had the potential to reduce stigma, raise awareness and increase access to services, one participant noted that there was still a lot more that should be done. Murungi noted that in many of the services that were offered (e.g., ride-share vehicles), there were few or no systems adapted to the abilities of persons with disabilities. Murungi also noted that some disabilities, such as autism spectrum disorder, were underrepresented. Five participants noted the importance of consulting with a diverse group of stakeholders before engaging in the technology design process. One participant, Kagenza, suggested the need for actively engaging persons with different abilities in the design process itself.

\subsection{Co-designing the Disability Ecosystem}

Using an adapted version of the Stakeholder Tokens method, participants created an ecosystem of diverse stakeholders with both linear and complex relationships within the disability ecosystem. 
4.2.1 The fluidity of core stakeholders. Participants were unanimous in their identification of persons with disabilities as core stakeholders within the ecosystem. This is consistent with the principles and strategies argued for in assistive technology literature [32, 65]. Other core stakeholders included: Families, Non-Governmental Organisations, Hospitals, Community Health Workers, Boda Boda Drivers, Bus Drivers, Taxi Drivers, Local City Authority, and Traffic Police. There were some differing opinions on whether the local technology start-up community was a core or periphery stakeholder. Only one participant argued their categorisation as core. This was because the participant had experience interacting with local technology hubs (i.e., co-working spaces that host local technologycentric events). Similarly, participants pointed out that in theory, they believe the Ministry of Health to be a core stakeholder. However, they failed to generate scenarios in which they had observed the Ministry of Health engaging with other parts of the ecosystem. This observation is particularly significant because of the position of influence the Ministry holds in terms of policy creation as well as its reputation for embracing innovative methods for service delivery $[13,50]$.

The relationships among the stakeholders also played a role in whether they were perceived as influential. A notable example of this are stakeholders that represented the transportation community (i.e., Boda Boda Drivers, Bus Drivers, and Taxi Drivers). In their example scenarios, participants justified their importance based on the role that they play as mobility providers. Secondly, Traffic Police also emerged as influential stakeholders in the ecosystem. Participants recounted scenarios where the traffic police acted as brokers of both security and safety.

\begin{abstract}
"Most of the persons with disabilities have trouble accessing the roads too so Traffic Police needs sensitization on how to handle these groups of people so that they can access transportation... Through the police, transport operators have to be sensitized on how to handle PWDs that are using various means of transport...." - Nassiwa

"... Traffic Police have an idea, especially on safety, transportation and also they can give us an advice...." - Kagenza
\end{abstract}

4.2.2 Traditionally influential, but on the periphery. Among the stakeholders who were thought to be on the periphery of the ecosystem were: Development Aid Partners; Ministry of Gender, Labour and Social Development; and Industry Corporate Social Responsibility Teams. Participants conceded that while Development Aid Partners and Industry Corporate Social Responsibility Teams were known for donations and hosting events (e.g, charity walks/runs), there were still more opportunities for them to foster stronger involvement and relationships beyond these events, a sentiment that has long been echoed in disability movements around the world [10].

The anomaly among this group of peripheral stakeholders is the Ministry of Gender, Labour and Social Development (MGLSD). The MGLSD is the official ministry responsible for the welfare and rights of persons with disabilities in Uganda [36]. There were only two connections drawn to this entity, one that indicated that activists lobby the MGLSD, and the other representing collaboration with the Ministry of Health. These two connections reveal MGLSD's participation with other stakeholders within the disability ecosystem. While this shows involvement, there is still opportunity for a direct connection between persons with disabilities and the MGLSD.

4.2.3 The promise of symbiotic partnerships. The Technology Startup Community starred at the intersection of three subgroups. Participants posited that the subgroup that included the Technology Startup Community and Government Ministries held an influential position regarding the implementation of inclusive policies. They reasoned that while it was up to the ministries to generate these policies, the Technology Startup Community should be creating products that can be used by people with a wide range of abilities anyway. The second grouping included the Boda Boda Drivers, Taxi Drivers, Bicycle Operators, Bus Drivers, and the Technology Startup Community. The theme surrounding this group was mainly a call for the creation of and advocacy for accessible transportation. Finally, the Technology Startup Community intersected with a third subgroup that also consisted of the Local City Authority and Non-Governmental Organisations. Participants noted that NonGovernmental Organisations have a history of working with people with disabilities in remote locations, while Local City Authorities have the resources to implement widespread change. Participants stated that a collaboration between these two entities and the Technology Startup Community would give voice and access to groups that are traditionally underrepresented.

4.2.4 A case of allies \& advocates. Allyship and advocacy were two of the connecting threads that tied together stakeholder representatives from the Social and Education spheres within the disability ecosystem. There were two notable examples of this. First, family members act as advocates for the well-being of their kin with a disability. Participants stated that Family Members interacted with Schoolteachers on behalf of their Family Member with a disability. A majority of the participants agreed that while activists fight for "disabled causes", they often do not have direct access to people with disabilities, often choosing instead to go through Non-Governmental Organisations, Family Members, and even in some instances Schoolteachers. In contrast, most participant experiences revealed the lack of interaction between persons with disabilities and non-familial members of the community (e.g., denoted by Pedestrians on our map). This provides additional weight to the need (mentioned in the earlier exercise) to create awareness through changing attitudes and thereby create allies.

4.2.5 Eliciting implicit values: human \& technological. The disability ecosystem designed emphasizes the values of inclusion, mobility and safety. These values were noted in the expression and examples surrounding stakeholders who are core in the ecosystem, i.e., the Person with a Disability, the Transportation Providers, and the Traffic Police. They stressed the need for the inclusion of persons with a diverse range of abilities. They also noted the importance of including diverse types of stakeholders as each stakeholder represented interests that were pertinent to the lived experiences of persons with disabilities. Examples included the transportation providers for mobility, the traffic police for safety, and schoolteachers for education. This demonstrates a shift in focus from the traditional clinical model (as observed in disability literature in countries like 
Uganda [34]) to a preference for the social model of disability. The underlying supposition around the value ascribed to technology is its capacity to do all, an assumption that was transferred and expected of the local Technology Startup Community. Katongole said that they expected the local technology start-up community to actively participate in solving challenges raised by persons with disabilities. For example, Katongole remarked on asking one of the local tech entrepreneurs to make for them a pair of new legs, after learning about the use of 3D prostheses in Uganda [44, 49].

“... us persons with disabilities we approach them with challenging issues. One time I went to someone and I was like could you please make for me legs... - Katongole"

\section{DISCUSSION}

\subsection{Disability Justice: Engaging the Lived Experience}

In our work, we demonstrate how the lived experiences of individuals with disabilities can be observed through stakeholder map generation and conversations about relationships (or the lack thereof) between different entities. Using this method, we were able to reveal unanticipated core stakeholders who regularly engaged with other members of the disability ecosystem for transportation and identify interesting relationships among known stakeholders. When seeking to change or update a complex system, it is critical to know and understand all of the key stakeholders in order to implement real, lasting changes with widespread engagement. These findings have two notable areas of impact. First, our observations are useful to HCI researchers who seek to create interventions that could be used in Global South and/or disability communities by relaying the values and relationships that are central to persons with disabilities in this context. Second, they underscore the importance of learning and understanding the lived experiences of persons with disabilities before designing technology, rather than elevating the design of technology as a solution or cure for their problems [21].

Our method and findings are aligned with broader movements worldwide that have advocated for the awareness and inclusion of persons of disabilities across various domains and geographies (e.g., politics and policy [10], design [24, 33, 39, 62, 65], etc.). Recently, these movements have given rise to the Disability Justice movement [20], which is particularly relevant to HCI scholars and practitioners who are interested in understanding the various ways in which technology may magnify marginalization. The Disability Justice movement advocates for persons with disabilities who exist at multiple intersections of social categorization and promotes understanding and elevating people's lived experiences. Through our work, we reiterate the importance of understanding and exploring these values when researching or practicing HCI.

\subsection{Positions and Perceptions of Power and Influence}

It is common practice for $\mathrm{HCI}$ for development practitioners and researchers to engage with multiple local stakeholders when developing, piloting, and evaluating systems [17, 22, 23, 37]. Often among these stakeholders are people who traditionally hold offices of power, such as Development Aid Partners, Industry Corporate
Social Responsibility Teams, and the Ministry of Gender, Labour and Social Development. Our work revealed evidence of nontraditional brokers of power within the disability ecosystem, such as Traffic Police and Transit Operators. This was a surprise because sought-out authorities on these topics typically include government ministries and development aid organisations. It is therefore important for scholars to understand the underlying power structures that exist within communities beyond the traditional hierarchies that are socially and even culturally observed in order to engage and impact an entire system.

Notably absent from this ecosystem are academic researchers, a fact that raises two questions: Is there a current lack of accessibility research for the Kampalan context or is accessibility research that is relevant to the local context not readily shared with persons with disabilities? This finding also raises an ethical concern that is pertinent to HCI researchers in both the Global North and the Global South regarding the distribution of research results. Many researchers would agree that participants who take part in the piloting and evaluation of HCI research should receive access to the resulting findings, but it is likely that fewer of us have considered how to transfer knowledge to a broader network of stakeholders within an ecosystem in order to ensure that our research can have impact. How can we best ensure that our work can positively benefit communities, particularly those that are typically underrepresented?

\subsection{Two Agendas: Physical Infrastructure \& Awareness}

This work identifies two agendas that emerged as important within the disability ecosystem: Accessible physical infrastructure and Awareness. While it may be out of the typical scope for HCI scholars to directly sponsor or change policy, there is an opportunity for researchers to engage in research that advocates for policy and social change. Specifically, researchers could focus on technological approaches to raising awareness of instances of inaccessible infrastructure [60], documenting and sharing experiences around conscious and unconscious bias, and discrimination and harassment of persons with disabilities. For example, both Project SideWalk [60] and Tiramisu [61,69] worked with local transportation providers to improve accessibility. Taking inspiration from this, strategic partnerships between researchers and mobility stakeholders, like ride-share companies in Kampala, present the unique position and opportunity to bring awareness to inaccessible physical infrastructure. This includes but is not limited to: lack of pavements, missing manhole covers, construction, broken pavements, and streets known for traffic violations during rush hour. Ride-share companies are in a unique position because their users travel far and wide. Therefore, users could be asked to crowdsource the information either about what they observed on their commute or about their locale. This information could then be aggregated and visualized in multiple formats (e.g., maps, text, etc.). Given the widespread prevalence of inaccessible physical infrastructure, this information could be used to inform users and policymakers about neighborhoods and high priority routes (e.g., [63]). Likewise, ride-share companies could harness the power of social media as an activism tool 
[28, 41, 42, 45] to raise awareness of problematic neighborhoods and advocate for the intervention of local city authorities.

Finally, Kampala is in the process of drafting policies to guide its transition into Smart City status [27, 30]. This provides an opportunity for researchers to engage in work that would expose ways in which the automation of existing unconscious cultural biases [34] may threaten the livelihoods of persons with disabilities. This is also an opportunity for research to have a direct impact on policy.

\section{CONCLUSION}

In this work, we have contributed to accessibility scholarship by describing the lived experiences of persons with disabilities in Kampala, Uganda. Using the stakeholder tokens method from the value design framework, we co-designed a stakeholder map of the transportation system with disability advocates. Our findings affirm the need for specific research emphases on inclusion, mobility, and safety. We also present a case for human-computer interaction scholars and practitioners to actively investigate the changing perceptions and positions of power that multiple stakeholders in the disability ecosystem hold, even beyond the realm of transportation. Lastly, we echo our participants' call to create awareness through engagement with other stakeholders, such as local ride-share service providers.

\section{ACKNOWLEDGMENTS}

We would like to thank our participants for taking time from their busy schedules to share their lived experiences with us. We would also like to thank Patricia Kahill, Pearl A. K. Byabashaija, Catherine Lubowa, Eva Muhanguzi, Joan Nkiriki, Vikram Kamath Cannanure, Judith Uchiduino, and the TBD Lab for their valuable insight throughout this study. Lastly, we thank our reviewers for their constructive feedback.

The contents of this paper were developed under a grant from the National Institute on Disability, Independent Living, and Rehabilitation Research (NIDILRR grant number 90REGE0007). NIDILRR is a Center within the Administration for Community Living (ACL), Department of Health and Human Services (HHS).

\section{REFERENCES}

[1] Syed Ishtiaque Ahmed, Nicola J. Bidwell, Himanshu Zade, Srihari H. Muralidhar, Anupama Dhareshwar, Baneen Karachiwala, Cedrick N. Tandong, and Jacki O'Neill. 2016. Peer-to-Peer in the Workplace: A View from the Road. In Proceedings of the 2016 CHI Conference on Human Factors in Computing Systems (San Jose, California, USA) (CHI '16). Association for Computing Machinery, New York, NY, USA, 5063-5075. https://doi.org/10.1145/2858036.2858393

[2] Sharon Eva Ahumuza, Joseph KB Matovu, John Bosco Ddamulira, and Florence Kyoheirwe Muhanguzi. 2014. Challenges in accessing sexual and reproductive health services by people with physical disabilities in Kampala, Uganda. Reproductive health 11, 1 (2014), 59. https://doi.org/10.1186/1742-4755-11-59

[3] Rehema Baguma. 2017. An Audit of Inclusive ICTs for Education in Uganda. In Proceedings of the 10th International Conference on Theory and Practice of Electronic Governance (New Delhi AA, India) (ICEGOV '17). Association for Computing Machinery, New York, NY, USA, 311-320. https://doi.org/10.1145/3047273.3047339

[4] Rehema Baguma, Tom Wanyama, PV Bommel, and Patrick Ogao. 2007. Web Accessibility in Uganda: A study of Webmaster perceptions. In proceedings of the 3rd Annual International Conference on Computing \& ICT Research (SREC'07). International Conference on ICT Research, Kampala, Uganda, 183-197.

[5] Giulia Barbareschi, Catherine Holloway, Katherine Arnold, Grace Magomere, Wycliffe Ambeyi Wetende, Gabriel Ngare, and Joyce Olenja. 2020. The Social Network: How People with Visual Impairment Use Mobile Phones in Kibera, Kenya. In Proceedings of the $2020 \mathrm{CHI}$ Conference on Human Factors in Computing Systems (Honolulu, HI, USA) (CHI '20). Association for Computing Machinery, New York, NY, USA, 1-15. https://doi.org/10.1145/3313831.3376658
[6] Giulia Barbareschi, Ben Oldfrey, Long Xin, Grace Nyachomba Magomere, Wycliffe Ambeyi Wetende, Carol Wanjira, Joyce Olenja, Victoria Austin, and Catherine Holloway. 2020. Bridging the Divide: Exploring the Use of Digital and Physical Technology to Aid Mobility Impaired People Living in an Informal Settlement. In The 22nd International ACM SIGACCESS Conference on Computers and Accessibility (Virtual Event, Greece) (ASSETS '20). Association for Computing Machinery, New York, NY, USA, Article 50, 13 pages. https://doi.org/10.1145/3373625.3417021

[7] Johan Borg, Anna Lindström, and Stig Larsson. 2009. Assistive technology in developing countries: national and international responsibilities to implement the Convention on the Rights of Persons with Disabilities. The Lancet 374, 9704 (2009), 1863-1865. https://doi.org/10.1016/S0140-6736(09)61872-9

[8] Virginia Braun and Victoria Clarke. 2006. Using thematic analysis in psychology. Qualitative research in psychology 3, 2 (2006), 77-101. https://doi.org/10.1191/ 1478088706qp063oa

[9] Patrick Businge. 2017. Disability and armed conflict: A quest for Africanising disability in Uganda. Disability and the Global South 3, 1 (2017), 816-842.

[10] James I Charlton. 2000. Nothing about us without us: Disability oppression and empowerment. Univ of California Press, Oakland, CA.

[11] Regis Chireshe, Edward Ntare Rutondoki, and Paul Ojwang. 2010. Perceptions of the availability and effectiveness of HIV/AIDS awareness and intervention programmes by people with disabilities in Uganda. SAHARA-7: fournal of Social Aspects of HIV/AIDS 7, 4 (2010), 17-23. https://doi.org/10.1080/17290376.2010. 9724973

[12] Rikke F. Dam and Teo Y. Siang. 2017. Define and Frame Your Design Challenge by Creating Your Point Of View and Ask "How Might We". Interaction Design Foundation. Retrieved August 6, 2020 from https://www.interaction-design.org/literature/article/define-and-frame-yourdesign-challenge-by-creating-your-point-of-view-and-ask-how-might-we

[13] eHealth Technical Working Group. 2016. Uganda National eHealth Policy. Ministry of Health. Retrieved August 8, 2020 from https://tmcg.co.ug/wp-content/uploads/ 2019/04/Uganda-National-eHealth-Policy-2016-.pdf

[14] Silvia Figueira, Michael Brew, Bryant Larsen, Pratyusha Joginipally, Sowmya Chandrashekarappa, and Ty Van Herweg. 2015. Text for a Ride, in Uganda. In Proceedings of the 4th International Conference on Information and Communication Technologies and Development ICTD (London, United Kingdom) (DEV '15). Association for Computing Machinery, New York, NY, USA, 75-76. https://doi.org/10.1145/2830629.2835220

[15] Batya Friedman and David G Hendry. 2019. Value sensitive design: Shaping technology with moral imagination. MIT Press, Cambridge, MA

[16] Dennis A Gioia. 2019. If I had a magic wand: Reflections on developing a systematic approach to qualitative research. Standing on the shoulders of giants: Traditions and innovations in research methodology 11 (2019), 27-37.

[17] Shikoh Gitau and Gary Marsden. 2009. Fair Partnerships - Working with NGOs. In Human-Computer Interaction - INTERACT 2009. Springer Berlin Heidelberg, Berlin, Heidelberg, 704-707.

[18] Shikoh Gitau, Paul Plantinga, and Kathleen Diga. 2010. ICTD research by Africans: Origins, interests, and impact. In Proceedings of the 4th International Conference on Information and Communication Technologies and Development ICTD. Association for Computing Machinery, New York, NY, USA, 13-16.

[19] Shikoh Gitau, Paul Plantinga, Kathleen Diga, and David Hutchful. 2011. African ICTD research (or the lack thereof). interactions 18, 4 (2011), 74-77. https: //doi.org/10.1145/1978822.1978837

[20] Aimi Hamraie. 2017. Building Access: Universal Design and the Politics of Disability. University of Minnesota Press, Minneapolis, MN. http://www.jstor.org/stable/ 10.5749/j.ctt1pwt79d

[21] Aimi Hamraie. 2020. Contra* Design with Bess Williamson and Elizabeth Guffey. Mapping Access. Retrieved September 7, 2020 from https://www.mapping-access.com/podcast/2020/6/22/contra-podcast-episode212-contrahistory-2-with-elizabeth-guffey-and-bess-williamson

[22] Melissa R. Ho, Emmanuel K. Owusu, and Paul M. Aoki. 2009. Claim Mobile: Engaging Conflicting Stakeholder Requirements in Healthcare in Uganda. In Proceedings of the $3 r d$ International Conference on Information and Communication Technologies and Development (Doha, Qatar) (ICTD'09). IEEE Press, New Jersy, US, 35-45.

[23] Melissa R Ho, Thomas N Smyth, Matthew Kam, and Andy Dearden. 2009. Humancomputer interaction for development: The past, present, and future. Information Technologies \& International Development 5, 4 (2009), 1-18.

[24] Catherine Holloway. 2019. Disability Interaction (DIX): A Manifesto. Interactions 26, 2 (Feb. 2019), 44-49. https://doi.org/10.1145/3310322

[25] Vaishnav Kameswaran, Jatin Gupta, Joyojeet Pal, Sile O’Modhrain, Tiffany C Veinot, Robin Brewer, Aakanksha Parameshwar, and Jacki O'Neill. 2018. 'We can go anywhere' Understanding Independence through a Case Study of Ride-hailing Use by People with Visual Impairments in metropolitan India. Proceedings of the ACM on Human-Computer Interaction 2, CSCW (2018), 1-24. https://doi.org/10. $1145 / 3274354$

[26] Vaishnav Kameswaran, Maulishree Pandey, Michigan Josh Guberman Hrishikesh Rao, and Michigan Sile O'Modhrain. 2019. Experiences of mobility in the Global South: Lessons from People with Visual Impairments in India. CHI'19 Workshops: 
Hacking Blind Navigation. Retrieved September 9, 2020 from http://vaikam. people.si.umich.edu/docs/HackingBlindNav.pdf

[27] Eronie Kamukama. 2019. Turning Kampala into a smart city. Monitor Publications. Retrieved 2019-09-16 from https://www.monitor.co.ug/Business/Prosper/ Turning-Kampala-into-a-smart-city-/688616-5122426-qke75t/index.html

[28] Ayesha Karamat and Ayesha Farooq. 2016. Emerging Role of Social Media in Political Activism: Perceptions and Practices. South Asian Studies (1026-678X) 31, 1 (2016), 381-396.

[29] Joseph Kasera, Jacki O’Neill, and Nicola J. Bidwell. 2016. Sociality, Tempo \& Flow: Learning from Namibian Ridesharing. In Proceedings of the First African Conference on Human Computer Interaction (Nairobi, Kenya) (AfriCHI'16). Association for Computing Machinery, New York, NY, USA, 36-47. https://doi.org/10.1145 2998581.2998582

[30] KCCA. 2019. ICT Department. KCCA. Retrieved 2019-09-16 from https://www. kcca.go.ug/ict

[31] Claire Kearney-Volpe, Amy Hurst, and Scott Fitzgerald. 2019. Blind Web Develop ment Training at Oysters and Pearls Technology Camp in Uganda. In Proceedings of the 16th Web For All 2019 Personalization - Personalizing the Web (San Francisco, CA, USA) (W4A '19). Association for Computing Machinery, New York, NY, USA Article 18, 10 pages. https://doi.org/10.1145/3315002.3317562

[32] Simeon Keates and P John Clarkson. 2003. Countering design exclusion: bridging the gap between usability and accessibility. Universal access in the information society 2, 3 (2003), 215-225. https://doi.org/10.1007/s10209-003-0059-5

[33] Simeon L. Keates and John Clarkson. 2004. Countering Design Exclusion: An Introduction to Inclusive Design. Springer, New York, USA

[34] Lynn Kirabo, Elizabeth J. Carter, and Aaron Steinfeld. 2020. "You Are Asking Me to Pay for My Legs": Exploring the Experiences, Perceptions, and Aspirations of Informal Public Transportation Users in Kampala and Kigali. In Proceedings of the 3rd ACM SIGCAS Conference on Computing and Sustainable Societies (Ecuador) (COMPASS '20). Association for Computing Machinery, New York, NY, USA, 136-147. https://doi.org/10.1145/3378393.3402269

[35] Jacqueline Klopp, Jackson Mutua, Dan Orwa, Peter Waiganjo, Adam White, and Sarah Williams. 2014. Towards a Standard for Paratransit Data: Lessons from Developing GTFS Data for Nairobi's Matatu System. Technical Report. Transportation Research Board.

[36] Pia Korpinen. 2009. Inclusion of People with Disabilities In Uganda. ILO/Irish Aid Partnership Programme. Retrieved August 8, 2020 from https://www.ilo.org/wcmsp5/groups/public/---ed_emp/---ifp_skills/ documents/publication/wcms_115099.pdf

[37] Neha Kumar and Nicola Dell. 2018. Towards Informed Practice in HCI for Development. Proceedings of the ACM on Human-Computer Interaction 2, CSCW (2018), 1-20. https://doi.org/10.1145/3274368

[38] Stanley Kwenda. 2010. Africa's disabled will not be forgotten. Africa Renewal 24, 1 (2010), 18-21. https://doi.org/10.18356/2d84257f-en

[39] Jonathan Lazar. 2007. Universal Usability: Designing Computer Interfaces for Diverse User Populations. John Wiley \& Sons, Inc., Hoboken, NJ, USA.

[40] Bridget Leadbeater. 2017. How disability studies and ecofeminist approaches shape research: exploring small-scale farmer perceptions of banana cultivation in the Lake Victoria region, Uganda. Disability and the Global South 2, 3 (2017), 752-776.

[41] Hanlin Li, Disha Bora, Sagar Salvi, and Erin Brady. 2018. Slacktivists or Activists? Identity Work in the Virtual Disability March. In Proceedings of the $2018 \mathrm{CHI}$ Conference on Human Factors in Computing Systems (Montreal QC, Canada) (CHI '18). Association for Computing Machinery, New York, NY, USA, 1-13. https://doi.org/10.1145/3173574.3173799

[42] Chris Linder, Jess S Myers, Colleen Riggle, and Marvette Lacy. 2016. From margins to mainstream: Social media as a tool for campus sexual violence activism Fournal of Diversity in Higher Education 9, 3 (2016), 231. https://doi.org/10.1037/ dhe 0000038

[43] Malcolm MacLachlan, Gubela Mji, Tsitsi Chataika, Margaret Wazakili, Andrew K Dube, Moses Mulumba, Boniface Massahm, Dagnachew Wakene, Frank Kallon, and Marcella Maughan. 2017. Facilitating disability inclusion in poverty reduction processes: group consensus perspectives from disability stakeholders in Uganda, Malawi, Ethiopia, and Sierra Leone. Disability and the Global South 1, 1 (2017), 107-127.

[44] Cory Mogk. 2015. A Repeatable 3D Printing Process for Low-Cost Prosthetics. Autodesk Research. Retrieved August 9, 2020 from https://autodeskresearch.typepad.com/blog/2015/03/repeatable-3d-printingprocess-for-low-cost-prosthetics.html

[45] Rosa Morris. 2013. 'Unjust, inhumane and highly inaccurate': the impact of changes to disability benefits and services-social media as a tool in research and activism. Disability \& Society 28, 5 (2013), 724-728.

[46] Joyce Nakatumba-Nabende, Benjamin Kanagwa, Florence Nameere Kivunike, and Michael Tuape. 2019. Evaluation of accessibility standards on Ugandan e-government websites. Electronic Government, An International fournal 15, 4 (2019), 355-371. http://doi.org/10.1504/EG.2019.102615

[47] Rose Nakibuule, Joseph Ssenyange, and John A. Quinn. 2013. Low Cost VideoBased Traffic Congestion Monitoring Using Phones as Sensors. In Proceedings of the 3rd ACM Symposium on Computing for Development (Bangalore, India) (ACM $D E V$ '13). Association for Computing Machinery, New York, NY, USA, Article 52, 2 pages. https://doi.org/10.1145/2442882.2442940

[48] Zahrah Nesbitt-Ahmed and Erika Fraser. 2017. Preliminary Impact Study. The use of safe boda for women's improved mobility and empowerment in Kampala. Technical Report. The Shell Foundation.

[49] Racheal Ninsiima. 2015. 3D prosthetic limbs come to Uganda. The Observer. Retrieved August 9, 2020 from https://www.observer.ug/news-headlines/361303d-prosthetic-limbs-come-to-uganda

[50] Ministry of Health. 2017. Uganda National eHealth Strategy: 2017 - 2021. Ministry of Health. Retrieved August 8, 2020 from https:/health.go.ug/sites/default/files/ National\%20e_Health\%20Strategy_0.pdf

[51] Uganda Bureau of Statistics. 2019. Persons With Disability Bridging The Gap Through Statistics. Uganda Bureau of Statistics, Kampala, Uganda.

[52] Ihudiya Finda Ogbonnaya-Ogburu, Angela DR Smith, Alexandra To, and Kentaro Toyama. 2020. Critical Race Theory for HCI. In Proceedings of the 2020 CHI Conference on Human Factors in Computing Systems. ACM Press, New York, NY, USA, 1-16. https://doi.org/10.1145/3313831.3376392

[53] Joyojeet Pal and Meera Lakshmanan. 2012. Assistive Technology and the Employment of People with Vision Impairments in India. In Proceedings of the Fifth International Conference on Information and Communication Technologies and Development (Atlanta, Georgia, USA) (ICTD '12). Association for Computing Machinery, New York, NY, USA, 307-317. https://doi.org/10.1145/2160673.2160711

[54] Joyojeet Pal, Manas Pradhan, Mihir Shah, and Rakesh Babu. 2011. Assistive Technology for Vision-Impairments: An agenda for the ICTD Community. In Proceedings of the 20th International Conference Companion on World Wide Web (Hyderabad, India) ( $W W W$ '11). Association for Computing Machinery, New York, NY, USA, 513-522. https://doi.org/10.1145/1963192.1963365

[55] Joyojeet Pal, Maura Youngman, Terence O'Neill, Priyank Chandra, and Cyprien Semushi. 2015. Gender and Accessibility in Rwanda and Malawi. In Proceedings of the Seventh International Conference on Information and Communication Technologies and Development (Singapore, Singapore) (ICTD '15). Association for Computing Machinery, New York, NY, USA, Article 5, 9 pages. https://doi.org/10.1145/2737856.2738020

[56] Dorian Peters, Susan Hansen, Jenny McMullan, Theresa Ardler, Janet Mooney, and Rafael A. Calvo. 2018. "Participation is Not Enough": Towards IndigenousLed Co-Design. In Proceedings of the 30th Australian Conference on ComputerHuman Interaction (Melbourne, Australia) (OzCHI '18). Association for Computing Machinery, New York, NY, USA, 97-101. https://doi.org/10.1145/3292147.3292204

[57] Brigitte Rohwerder. 2018. Assistive technologies in developing countries. K4D Helpdesk Report. Institute of Development Studies, Brighton, UK.

[58] Simon Saddier and Alex Johnson. 2018. Understanding the Operational Characteristics of Paratransit Services in Accra, Ghana: A Case Study. Transportation Research Record: Journal of the Transportation Research Board. Retrieved September 9, 2020 from https://rid.trb.org/view/1496820

[59] Simon Saddier, Zachary Patterson, Alex Johnson, and Natalie Wiseman. 2017. Fickle or Flexible? Assessing Paratransit Reliability with Smartphones in Accra, Ghana. Transportation Research Record: fournal of the Transportation Research Board 4, 2650 (2017), 9-17. https://doi.org/10.3141/2650-02

[60] Manaswi Saha, Michael Saugstad, Hanuma Teja Maddali, Aileen Zeng, Ryan Holland, Steven Bower, Aditya Dash, Sage Chen, Anthony Li, Kotaro Hara, and Jon Froehlich. 2019. Project Sidewalk: A Web-Based Crowdsourcing Tool for Collecting Sidewalk Accessibility Data At Scale. In Proceedings of the 2019 CHI Conference on Human Factors in Computing Systems (Glasgow, Scotland Uk) (CHI '19). Association for Computing Machinery, New York, NY, USA, 1-14. https://doi.org/10.1145/3290605.3300292

[61] Aaron Steinfeld, Leslie Bloomfield, Sarah Amick, Yun Huang, Will Odom, Qian Yang, and John Zimmerman. 2019. Increasing access to transit: localized mobile information. Journal of urban technology 26, 3 (2019), 45-64. https://doi.org/10. 1080/10630732.2019.1614896

[62] Edward Steinfeld and Jordana Maisel. 2012. Universal Design: Creating Inclusive Environments. John Wiley \& Sons, Inc., Hoboken, NJ, USA.

[63] Elizabeth J Traut and Aaron Steinfeld. 2019. Identifying commonly used and potentially unsafe transit transfers with crowdsourcing. Transportation research part A: policy and practice 122 (2019), 99-111. https://doi.org/10.1016/j.tra.2019. 02.005

[64] Greg Walsh. 2018. Towards Equity and Equality in American Co-Design: A Case Study. In Proceedings of the 17th ACM Conference on Interaction Design and Children (Trondheim, Norway) (IDC '18). Association for Computing Machinery, New York, NY, USA, 434-440. https://doi.org/10.1145/3202185.3202768

[65] Jacob O. Wobbrock, Shaun K. Kane, Krzysztof Z. Gajos, Susumu Harada, and Jon Froehlich. 2011. Ability-Based Design: Concept, Principles and Examples. ACM Trans. Access. Comput. 3, 3, Article 9 (April 2011), 27 pages. https://doi.org/10. 1145/1952383.1952384

[66] Daisy Yoo. 2017. Stakeholder Tokens: A Constructive Method for Value Sensitive Design Stakeholder Analysis. In Proceedings of the 2017 ACM Conference Companion Publication on Designing Interactive Systems. ACM Press, New York, NY, 280-284. https://doi.org/10.1145/3064857.3079161 
[67] Daisy Yoo, John Zimmerman, and Tad Hirsch. 2013. Probing Bus Stop for Insights on Transit Co-Design. In Proceedings of the SIGCHI Conference on Human Factors in Computing Systems (Paris, France) (CHI '13). Association for Computing Machinery, New York, NY, USA, 409-418. https://doi.org/10.1145/2470654.2470714

[68] Daisy Yoo, John Zimmerman, Aaron Steinfeld, and Anthony Tomasic. 2010. Understanding the Space for Co-Design in Riders' Interactions with a Transit Service. In Proceedings of the SIGCHI Conference on Human Factors in Computing Systems (Atlanta, Georgia, USA) (CHI '10). Association for Computing Machinery, New
York, NY, USA, 1797-1806. https://doi.org/10.1145/1753326.1753596

[69] John Zimmerman, Anthony Tomasic, Charles Garrod, Daisy Yoo, Chaya Hiruncharoenvate, Rafae Aziz, Nikhil Ravi Thiruvengadam, Yun Huang, and Aaron Steinfeld. 2011. Field Trial of Tiramisu: Crowd-Sourcing Bus Arrival Times to Spur Co-Design. In Proceedings of the SIGCHI Conference on Human Factors in Computing Systems (Vancouver, BC, Canada) (CHI '11). Association for Computing Machinery, New York, NY, USA, 1677-1686. https://doi.org/10.1145/1978942. 1979187 